\title{
Iron deficiency anaemia in patients with rheumatic disease receiving non-steroidal anti-inflammatory drugs: the role of upper gastrointestinal lesions
}

\author{
R Upadhyay, H I Torley, A W McKinlay, R D Sturrock, R I Russell
}

\begin{abstract}
Upper gastrointestinal lesions associated with non-steroidal anti-inflammatory drug (NSAID) treatment are commonly implicated as the cause for iron deficiency anaemia in patients with rheumatic diseases. Such patients, however, may also have other causes for iron deficiency, including blood loss from the intestine. One hundred and four patients (mean age 58 years; male 21, female 83; smokers 14) with rheumatic disease (rheumatoid 91, others 13) and absent bone marrow iron stores (mean haemoglobin $83 \mathrm{~g} / \mathrm{l}$ ) were examined. At endoscopy 47 of $104(45 \%)$ had upper gastrointestinal lesions (oesophageal ulcer 4, gastric ulcer 25 , gastric erosion 13 , duodenal ulcer 4, gastric ulcer and duodenal ulcer 1). Endoscopic healing was assessed in 23 patients with upper gastrointestinal lesions. Eighteen of $23(78 \%)$ lesions healed with treatment. An improvement of anaemia occurred in 10 of $18(56 \%)$ patients with healed lesions. Twenty three of $104(22 \%)$ patients had dyspeptic symptoms. Ten of 23 (43\%) patients with dyspepsia had an upper gastrointestinal lesion as compared with $\mathbf{3 0}$ of $81(37 \%)$ patients without dyspepsia. A faecal occult blood test result was available in 53 patients. Of these, 13 were positive while 40 were negative. An upper gastrointestinal lesion was present in seven of $13(54 \%)$ patients positive for the faecal occult blood test as compared with 14 of 40 (35\%) negative for the test. Thus upper gastrointestinal lesions have previously been overestimated as the cause of iron deficiency anaemia in patients receiving NSAIDs. A positive faecal occult blood test or the presence of dyspepsia is not associated with upper gastrointestinal lesions in such patients.
\end{abstract}

Gastroenterology Unit, Royal Infirmary, Glasgow G31 2ER $R$ Upadhyay A W McKinlay R I Russell

Centre for Rheumatic Diseases,

Royal Infirmary,

Glasgow G31 2ER

H I Torley

R D Sturrock

Correspondence to Dr R Upadhyay, Gastroenterology Unit,

Glasgow Royal Infirmary,

Alexandra Parade,

Glasgow G31 2ER.

Accepted for publication

25 July 1989
Once iron deficiency anaemia has been diagnosed, a cause must be identified. In the absence of dietary deficiency, blood loss is a common cause of iron depletion. There is a high prevalence of upper gastrointestinal lesions associated with NSAID treatment in rheumatic patients. ${ }^{5}$ These lesions are commonly presumed to be the cause of anaemia in such patients. Recent studies, however, have shown that intestinal and colonic inflammation also occur commonly in patients with rheumatic diseases receiving NSAIDs. ${ }^{78}$ Such lesions may also lead to blood and protein loss from the gut ${ }^{9}$ and consequently cause iron deficiency. The aim of the study was to examine the role of upper gastrointestinal lesions in iron deficiency anaemia of rheumatic patients receiving NSAIDs. We initially determined the prevalence of upper gastrointestinal lesions in patients with absent iron stores, and then examined the effect of healing the lesions on the outcome of the anaemia. Thus we attempted to establish a cause and effect relation between the upper gastrointestinal lesions and the anaemia. We also evaluated whether the presence of dyspepsia or a positive faecal occult blood test may be a guide to the presence of upper gastrointestinal lesions.

\section{Patients and methods}

A retrospective survey was made of patients with rheumatic disease who had absent iron stores on bone marrow aspirate. Patients were identified from haematology records of all bone marrow aspirations performed between 1984 and 1988. Additional patients were identified from upper gastrointestinal endoscopy referrals during the same period, in which iron deficiency anaemia was recorded as one of the indications. Patients were only included if they had been receiving NSAIDs for more than three months and had undergone at least one endoscopic examination of the upper gastrointestinal tract.

One hundred and four patients fulfilled the criteria. Their case records were examined for information about dyspeptic symptoms, smoking habit, drug ingestion, previous peptic ulceration, gastric surgery, and faecal occult blood test results. The haemoglobin, erythrocyte sedimentation rate, platelet count, mean corpuscular volume, serum vitamin $B_{12}$ and folate, renal function tests, and all gastrointestinal tests were also recorded.

All bone marrow examinations and most endoscopies were carried out during hospital admission. All endoscopies during the period of the study were performed independently by two operators and reported in the standardised format normally used in the gastroenterology unit. A lesion was defined as a macroscopic breach of mucosa in the oesophagus, stomach, or duodenum. This included lesions such as 
erosions and ulcers but excluded abnormalities such as erythema or intramucosal haemorrhage, the significance of which is controversial.

A faecal occult blood test had been performed on the subjects as inpatients using the Haemoccult test on three consecutive days. The test was considered positive if any of the three samples were positive. Where endoscopic healing was confirmed the haemoglobin concentration was followed up for at least three months after healing. An improvement of anaemia in these patients was defined as a sustained rise in haemoglobin $>15 \mathrm{~g} / \mathrm{l}$.

\section{Results}

Table 1 shows the demographic details of the 104 patients included in the study. Forty seven of $104(45 \%)$ patients had endoscopic evidence of an upper gastrointestinal lesion. Gastric lesions were the commonest, being present in 39 of $47(83 \%)$ patients with lesions. Table 2 shows details of the endoscopic findings. The healing of upper gastrointestinal lesions was assessed by endoscopy in 23 patients. In 18 of $23(78 \%)$ patients the lesion had healed with $\mathrm{H} 2$ antagonist treatment despite continuing NSAID treatment. A sustained improvement of anaemia occurred in 10 of $18(56 \%)$ patients with endoscopically healed lesions.

Twenty three of $104(22 \%)$ patients had dyspeptic symptoms while $81(78 \%)$ patients were asymptomatic. Ten of $23(43 \%)$ patients with dyspepsia had an upper gastrointestinal lesion as compared with 30 of 81 (37\%) patients

Table 1: Demographic details of the rheumatic patients with iron deficiency anaemia

\begin{tabular}{lc}
\hline Sex & 21 \\
Male & 83 \\
Female & 58 \\
Age (years) & $23-81$ \\
Mean & 91 \\
Range & 5 \\
Arthritis & 4 \\
Rheumatoid & 3 \\
Psoriatic & 1 \\
Seronegative polyarthropathy & $14(13)$ \\
Ankylosing spondylitis & $90(87)$ \\
Systemic lupus erythematosus & 104 \\
Smoking (No (\%)) & $29(28)$ \\
Smokers & $4(4)$ \\
Non-smokers & 21 \\
Drugs & \\
NSAID: & $83(1)$ \\
Second line drug (No (\%)) & \\
Prednisolone (No (\%)) & \\
Antiulcer drug & H2 antagonist \\
Anaemia & \\
Mean (SEM) haemoglobin (g/l) & \\
\hline
\end{tabular}

${ }^{*}$ NSAID $=$ non-steroidal anti-inflammatory drug.

Table 2: Endoscopic findings in the rheumatic patients with iron deficiency anaemia

\begin{tabular}{lc}
\hline Endoscopic findings & $\begin{array}{l}\text { Number of } \\
\text { patients }\end{array}$ \\
\hline Normal & 37 \\
Gastritis/duodenitis & 11 \\
Intramucosal haemorrhages & 4 \\
Oesophageal ulcer & 4 \\
Gastric erosions & 13 \\
Gastric ulcers & 25 \\
Duodenal ulcer & 4 \\
Gastric ulcer and duodenal ulcer & 1 \\
Atropy (gastric) & 1 \\
Polyps (gastric reg) & 1 \\
Gastric surgery & 3 \\
\hline
\end{tabular}

without dyspepsia $\left(\chi^{2}=0 \cdot 10 ; \mathrm{p}>0 \cdot 7 ; \mathrm{NS}\right)$ (fig 1). Faecal occult blood test results were available in 53 patients. Of these, 13 were positive and 40 negative. Seven of $13(54 \%)$ of patients positive for the faecal occult blood test had upper gastrointestinal lesions as compared with 14 of $40(35 \%)$ negative for the test (fig 2). This, however, was not significant $\left(\chi^{2}=0 \cdot 77 ; p=0 \cdot 4\right.$; NS).

Fourteen of the 104 patients were smokers. Five of the $14(36 \%)$ had dyspeptic symptoms. Six of the $14(43 \%)$ smokers had upper gastrointestinal lesions as compared with 28 of the 90

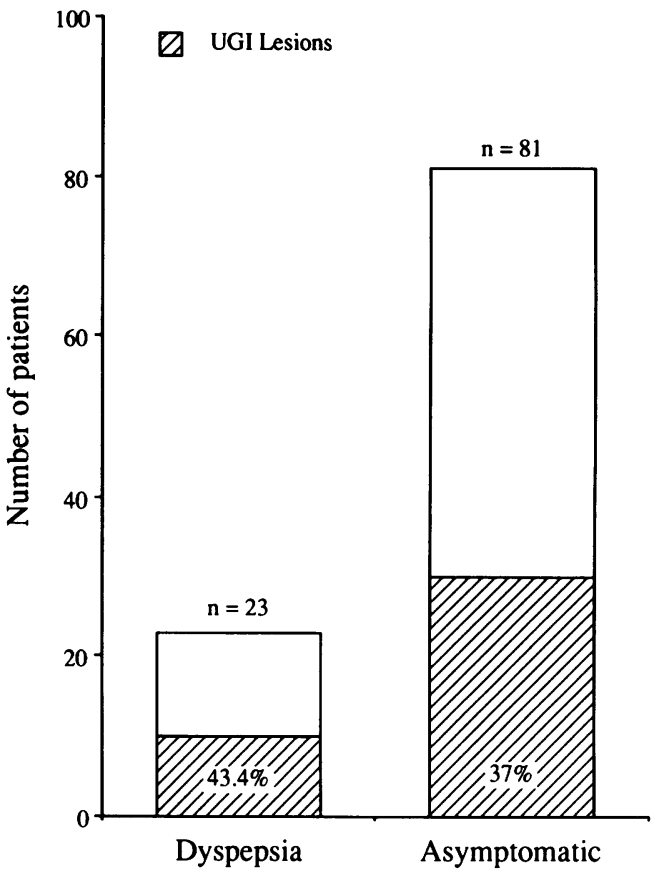

Figure 1: Prevalence of upper gastrointestinal (UGI) lesions in patients with and without dyspeptic symptoms.

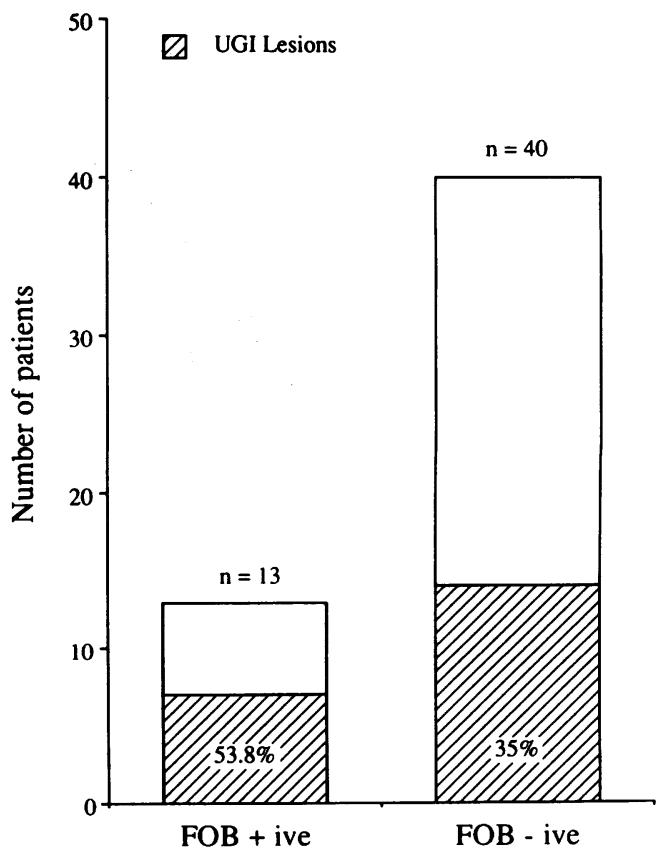

Figure 2: Prevalence of upper gastrointestinal (UGI) lesions in patients with positive or negative faecal occult blood (FOB) test. 
(31\%) non-smokers $(p=0 \cdot 17) ; N S)$. Twenty one patients were receiving regular $\mathrm{H} 2$ antagonist treatment (dyspepsia 10, previous peptic ulcer 9, oesophagitis 1 , and recent history of melaena 1). Nine of the $21(43 \%)$ patients receiving $\mathrm{H} 2$ antagonist treatment had upper gastrointestinal lesions. Five of the nine patients receiving $\mathrm{H} 2$ antagonists for previous peptic ulceration had upper gastrointestinal lesions. Thirty nine patients had double contrast barium enema examination. Of these, 23 were normal, 14 had diverticular disease, and two showed filling defects in the colon. Both patients with filling defects had a normal colonoscopy.

\section{Discussion}

This study shows that upper gastrointestinal lesions are present in less than half of patients receiving NSAIDs who have absent bone marrow iron stores. In addition, the anaemia improved in only $10 / 18(56 \%)$ patients with endoscopically healed upper gastrointestinal lesions. This implies that the role of such lesions as a cause of iron deficiency anaemia in rheumatic patients might have been overestimated previously. In addition, the presence of upper gastrointestinal lesions should not necessarily be accepted as the sole cause of anaemia. The interpretation that the improvement in anaemia after lesion healing implies a cause and effect relation, however, may be confounded by other factors. Thus blood transfusion, compliance with oral iron treatment, and disease activity may influence the haemoglobin concentration during the follow up. All but one patient in whom endoscopic healing was assessed had received a blood transfusion. This would not, therefore, have influenced our results. Whether the haemoglobin concentration was influenced by compliance with oral iron treatment or disease activity remains unknown. The cause and effect relation between the upper gastrointestinal lesions and the anaemia can only be accepted within these limitations.

A previous study ${ }^{10}$ has also reported a similar prevalence of upper gastrointestinal lesions. All patients with anaemia were included, however, irrespective of the type. In addition, lesions of the oesophagus were excluded. The association of oesophageal lesions with NSAID treatment is recognised $^{11} 12$ and may be an important cause of anaemia in such patients. Two of the four patients with oesophageal ulceration in our study had episodes of haematemesis related to the lesion.

There was only a small number of smokers (14 of 104) among our patients. It has been noted that the rate at which smoking is stopped rises with age, ${ }^{13}$ which may explain the small number of smokers in our group. Alternatively, this may be related to their chronic disease and the repeated instructions to stop smoking, during their clinic attendance. Smoking was not associated with either dyspepsia or upper gastrointestinal lesions in our patients. The study also confirms that upper gastrointestinal lesions may be satisfactorily healed with $\mathrm{H} 2$ antagonist treatment despite continuing NSAID treatment. ${ }^{14}$ Interestingly, five of the nine patients who were taking $\mathrm{H} 2$ antagonists as maintenance treatment following previous peptic ulcer disease had upper gastrointestinal lesions. All these patients were free from dyspeptic symptoms. This clearly shows the problem of asymptomatic recurrence of peptic ulcer disease while they were receiving maintenance $\mathrm{H} 2$ antagonist treatment.

Barium enema examination was not helpful in identifying the source of blood loss. Patients with significant ileocolonic inflammation may have normal barium enema. Alternatively, the significance of diverticulosis as the cause for anaemia can be debated. In our study the two patients with filling defects on barium enema turned out to be false positives. It has been reported that $20 \%$ of patients positive for the faecal occult blood test taking NSAIDs may have colonic neoplasia. ${ }^{16}$ Colonoscopy is better than barium enema in diagnosing lesions in the colon ${ }^{17}$ and should be the preferred method of investigation. Although the needless submission of frail patients or those with severe joint disease to invasive investigations is to be avoided, we believe that colonoscopy should be used wherever possible.

Our survey also suggests a reluctance by clinicians to undertake small bowel investigations. Of six patients with low serum vitamin $B_{12}$ and normal upper gastrointestinal endoscopy results, only two had been referred for small bowel radiology (both normal). The lack of a simple and specific test to identify small bowel inflammation and ulceration may be an important factor mediating against the wider uptake of such investigations. Small bowel radiology can be time consuming and may be poorly tolerated by patients with severe, painful joint disease. The radioisotope techniques require multiple scans ${ }^{9}$ and interpretation of clinical significance can be debated. Enteroscopy ${ }^{18}$ awaits further evaluation. Even when a diagnosis of an NSAID associated bowel lesion is made, there is no specific treatment apart from withdrawing the drug. The tendency, therefore, from a practical standpoint might have been to presume small intestinal blood loss in patients with no other obvious cause for iron deficiency and who have normal upper gastrointestinal endoscopy and colonic investigation. Withdrawal or change of NSAID was attempted in a number of such cases. The problem, however, is that with such an approach it is possible that some other small bowel diagnosis may be missed.

No correlation between the presence of dyspepsia and upper gastrointestinal lesions could be shown in this study. Although this correlation is well known in patients receiving NSAIDs, ${ }^{5} 6$ it has not been reported in patients with iron deficiency before. In such a group of patients, therefore, the presence or absence of dyspepsia should not decide the plan of investigation.

Our study also confirms the previously reported poor correlation between a positive faecal occult blood test and upper gastrointestinal lesions. ${ }^{19}$ It also shows that only a small number of our patients were positive for this test, probably because the Haemoccult card test 
was used, which is insensitive to blood loss caused by NSAIDs. ${ }^{20}$

In conclusion, our study indicates that upper gastrointestinal lesions have been overestimated as the cause of iron deficiency anaemia in patients receiving NSAIDs. Perhaps intestinal blood loss may be a more important cause of anaemia in such patients.

1 Mowat A G. Haematologic abnormalities in rheumatoid arthritis. Semin Arthritis Rheum 1971; 1: 195-219.

2 Owen E T, Lawson A A H. Nature of anaemia in rheumatoid arthritis. VI. Metabolism of endogenous iron. Ann Rheum Dis 1966; 25: 547-52.

3 Bennett R M, Holts P J L, Lewis S M. Role of recituloendothelial system in the anaemia of rheumatoid arthritis. Ann Rheum Dis 1974; 33: 147-52.

4 Zoma A, Hambley H, Sturrock R D. Prediction of marrow iron stores and serum ferritin levels. $\mathrm{Br} \mathcal{F}$ Rheumatol 1987 26 (suppl 2): 36

5 Caruso I, Bianchi Porro. Gastroscopic evaluation of antiinflammatory drugs. $\mathrm{Br}$ Med $\mathcal{f}$ 1980; 280: 75-8.

6 Larkai E N, Lacey Smith J, Lidsky M D, Graham D Y. Gastroduodenal mucosa and dyspeptic symptoms in arthritis patients during chronic nonsteroidal anti-inflammatory use. patients during chronic nonsteroidal antiont

7 Bjarnason I, Zanelli G, Smith T, et al. Nonsteroidal antiinflammatory drug-induced intestinal inflammation in antiinflammatory drug-induced intestinal

8 De Vos M, Cuvelier C, Mielants H, Veys E, Barbier F, Elewaut A. Ileocolonoscopy in seronegative spondylarthropathy. Gastroenterology 1989; 96: 339-44.
9 Bjarnason I, Prouse P, Smith T, et al. Blood and protein loss via small-intestinal inflammation induced by non-steroida anti-inflammatory drugs. Lancet 1987; ii: $711-4$.

10 Doube A, Collins A J. Anaemia in patients with arthritis: Are simple investigations helpful? $B r \mathcal{F}$ Rheumatol 1988; 27: 303-5.

11 Heller S R, Fellows I W, Ogilvie A L, Atkinson M. Nonsteroidal anti-inflammatory drugs and benign oesophageal stricture. Br Med f 1982; 285: 167-8.

12 Shallcross T, Wyatt J I, Heatly R V. Oesophageal ulceration, NSAIDs and hiatus hernia. Gut 1989; 30: A728.

13 Kabat G C, Wynder E L. Determinants of quitting smoking. Am 7 Public Health 1987; 77: 1301-5.

14 Croker J R, Cotton P B, Boyle A C, Kinsella P. Cimetidine for peptic ulceration in patients with arthritis. Ann Rheum Dis 1980; 39: 275-8.

15 Molchow-Moller A, Mannichi C, Anderson J R, et al. Does cessation of NSAID therapy influence the healing of peptic ulcer in rheumatoid patients? Scand $\mathcal{F}$ Gastroenterol 1985; 20 ulcer in rheumappl 113): 53 .
(supe

16 Pye G, Ballantyne K C, Armitage N C, Hardcastle J D. Influence of non-steroidal anti-inflammatory drugs on the outcome of faecal occult-blood tests in screening for colorectal cancer. $\mathrm{Br} \mathrm{Med} \mathrm{J} \mathrm{1987;} \mathrm{294:} \mathrm{1510-1.}$

17 Aldridge M C, Sim A J W. Colonoscopy findings in symptomatic patients without X-ray evidence of colonic neoplasms. Lancet 1986; ii: $833-4$.

18 Lewis B S, Waye J D. Chronic gastrointestinal bleeding of obscure origin: role of small bowel enteroscopy Gastroenterology 1988; 94: 1117-20.

19 Collins A J, Du Toit J. Upper gastrointestinal findings and faecal occult blood in patients with rheumatic diseases taking nonsteroidal anti-inflammatory drugs. Br 7 Rheumatol 1987; 26: 295-8.

20 Bahrt K M, Karman L Y, Nashel D J. Significance of a positive test for faecal occult blood in stools of patients taking anti-inflammatory drugs. Arch Intern Med 1984; 144: 2165-6. 